\title{
MINIMAL $A$-SETS, INFINITE ORBITS, AND FIXED ELEMENTS
}

\author{
G. E. SCHWEIGERT
}

Throughout this note $S$ denotes a semi-locally-connected continuum ${ }^{1}$ and $T(S)=S$ an on to-homeomorphism. If $E$ is a set of $S$ such that $T^{k}(E)=E$ for some positive integer $k$, then $E$ has a finite period, otherwise the period for $E$ is infinite. The set of all images of $E$ under $T$ and its inverse $T^{-1}$ is said to be the orbit of $E$. If $E$ is of period $k=1$, then $E$ is invariant under $T$. We investigate the least invariant $A$-set which contains the orbit of a cyclic element $E$, when the period and orbit for $E$ are infinite. This work occupies an intermediate position between a previous paper, ${ }^{2}$ wherein certain general results are directed toward the study of finite orbits, and the problem of the action of $T(S)=S$ in general. We follow here the spirit of the work of Ayres begun in his paper, On transformations having periodic properties, Fund. Math. vol. 33 (1939) pp. 95-103. With Ayres we denote the unique cyclic chain between two cyclic elements $E$ and $D$ by $C(E, D)$.

THEOREM $^{3}$ A. If $E$ is any cyclic element of $S$ with an infinite period and $B$ is the least (invariant $\left.{ }^{4}\right) A$-set containing the orbit of $E$ then one of the following cases must occur:

(a) $B$ contains exactly one fixed element $F$. In this case if $B$ is cyclic then $E$ is a single point which is a cut point of $S$ lying in $B$. The set $S-B$ then has infinitely many distinct components bounded by images of $E$. (Of course it may also have other components not bounded by these images.) If $B$ is not cyclic then for any element $E^{\prime}$ of the orbit of $E$ the set $B$ is the closure of the orbit of the cyclic chain $C\left(E^{\prime}, F\right)$.

(b) B contains exactly two fixed elements $X$ and $Y$. In this case $X$

Presented to the Society, April 23, 1943; received by the editors March 15, 1943.

${ }^{1}$ See G. T. Whyburn, Analytic topology, Amer. Math. Soc. Colloquium Publications vol. 28, 1942, pp. 64-98. It is assumed that the reader is familiar with the cyclic element theory and the general terminology of this publication. We refer to this book hereafter as ATW with the numbers for theorems in parentheses.

${ }^{2}$ Fixed elements and periodic types for homeomorphism on s.l.c. continua, Proc. Nat. Acad. Sci. U. S. A. vol. 29 (1942) p. 52. To appear in full in the Amer. J. Math. under the same title. This paper will be referred to as F; the numbers in parentheses refer to theorems of the complete paper.

${ }^{3}$ Compare $F(2.4)$. Each theorem of this paper has as a corollary the special case in which $S$ is a dendrite. The form used in stating Theorem $\mathrm{A}$ and the general nature of the proof are at the suggestion of the referee.

4 The least $A$-set containing an invariant set is invariant. 
and $Y$ are end points of $B$ and all elements of $B$ except $X$ and $Y$ have infinite periods. Furthermore:

(1) if $B=C(X, Y)$ then the images $T^{n}(E)$ are linearly ordered on $C(X, Y)$ as they occur under $T$ for $n$ in the range of all integers.

(2) if $B \neq C(X, Y)$ then $C(X, Y)$ contains an element $E^{*}$ satisfying (1) and such that $C(X, Y)$ is the minimal invariant $A$-set in $S$ containing the orbit of $E^{*}$. Moreover, if $E_{n}$ and $E_{n}^{*}$ denote the nth images of $E$ and $E^{*}$, respectively, under $T$, then the cyclic chain $C\left(E_{n}, E_{n}^{*}\right)$ in $B$ contains the component of $B-E_{n}{ }^{*}$ which has $E_{n}$ as a subset of its closure.

Proof. By a theorem of Ayres ${ }^{5} B$ must con tain at least one invariant cyclic element. If it contains exactly one such invariant element $F$ then (a) holds and the results in this case are a very easy exercise for the reader. Otherwise, $B$ must contain at least two invariant cyclic elements and we denote any two such elements by $X$ and $Y$. Thus the cyclic chain $C(X, Y)$ is invariant under $T$ and every element which is not fixed has an infinite orbit. Now if $C(X, Y)$ contains an element of the orbit of $E$ then it must contain the entire orbit and we have $B=C(X, Y)$. It follows at once that $C(X, Y)$ can contain no other invariant element by the fact that $B$ is minimal and $X$ and $Y$ were arbitrary. From the minimal character of $B$ we see that $X$ and $Y$ are end points of $B$, and (1) holds. In the event that no element in the orbit of $E$ is in $B$ there is again no third invariant element in $B$. Such an element $Z$ would imply that $Z \subset \bar{K}$, where $K$ is some component of $B-C(X, Y)$. From the minimal property of $B$ it follows that $\bar{K}$ must contain an element of the orbit of $E$. Thus the entire orbit of $E$ must lie in both of the $S$ cyclic chains $C(Z, X)$ and $C(Z, Y)$. This easily gives an $A$-set which contains the orbit of $E$ and is a proper subset of $B$, contrary to the definition of this set. Thus $B$ contains exactly two invariant cyclic elements $X$ and $Y$. Let $K(E)$ denote the component of $B-C(X, Y)$ having $E$ as a subset of its closure, and $p$ the boundary point of this component. Then if $E^{*}$ is the minimal cyclic element of $C(X, Y)$ containing $p$ we see that $E^{*}$ has an infinite orbit. This gives us (2) without difficulty and completes the proof of Theorem A.

The property mentioned below is one of five previously shown ${ }^{6}$ to be equivalent.

Corollary. Let $E$ be any cyclic element in $S$ such that $E$ and $E_{1}=T(E)$ are distinct. In order to insure that $C\left(E, E_{1}\right)$ has the third property of Ayres-contains exactly one invariant element $Z$-it is suffi-

${ }^{5}$ ATW (2.51) p. 242.

${ }^{6} \mathrm{~F}(2.6)$. 
cient (and necessary) to assume that an invariant element in $C\left(E, E_{1}\right)$ exists provided $E$ has an infinite period. There is no assumption when $E$ has finite period.

Proof. If the period for $E$ is infinite this follows from Theorem A. If $E$ has finite period, the least $A$-set $M$ containing the orbit of $E$ also contains a fixed cyclic element $Z$ by virtue of the theorem of Ayres. It is readily shown that: (1) $M$ is the finite orbit of $C\left(E, E_{1}\right)$, (2) $Z \subset C\left(E, E_{1}\right),(3) Z$ is unique.

If $M$ is a sum of cyclic elements of $S$ and each element of $M$ has a finite period then $T$ is said to be elementwise periodic on $M$. The case $M=S-L$, where $L$ is the set of all end points of $S$, has received considerable attention $;^{7}$ however, the question treated below awaited a study of infinite orbits.

THEOREM B. If $T(S)=S$ is elementwise periodic on $S-L$ and $p \in L$ has an infinite orbit, then the least $A$-set $M$ containing the orbit of $p$ has a unique invariant element ("hub") E, and the cyclic element hyperspace of $M$ is structurally similar to an n-adic tree. ${ }^{8}$

PRoof. The set $M$ is invariant and contains the closure of the orbit of $p$. In view of the density of the elements of finite period and Theorem $\mathrm{A}$ it is evident that $M$ contains a unique invariant element $E$. Let $k$ be the least positive value of the period function on $M-E$ and $I(k)$ the set of all cyclic elements of $M$ fixed under $T^{k}$. It follows ${ }^{9}$ that $I(k)$ is an $A$-set. Furthermore the minimal property for $M$ insures that $M-E$ is a component-orbit, the finite orbit of some component $K$ of $M-E$. We select one particular $K$ and consider $\bar{K}=K+q$, with $q$ a cut point of $M$ on $E$. (Note: $T(q) \neq q$ although $T^{2}(q)=q$ is possible.) Let $q \in C$, where $C$ is a true cyclic element of $K$, then $q \in I(k)$ implies $C \subset I(k)$. If $C$ does not exist, $q$ is an end point of $K$, and hence, by a previous theorem, ${ }^{10}$ we again get $I(k) \cdot K \neq 0$. Thus, in any case, there is a node $X$ of $I(k)$ in $\bar{K}$ by virtue of well known results. ${ }^{11}$ Finally $C(X, E) \subset I(k)$ also follows from a previous theorem. ${ }^{12}$ We now let $N=I(k) \cdot \bar{K}$ and investigate the nature of this $A$-set and its complement. If $R$ is a component of $\bar{K}-N, F(R)=x$,

${ }^{7} \mathrm{~F}(4.2)$ and ATW (4.6) p. 248.

${ }^{8}$ Tree $=$ dendrite of ATW. For the dyadic tree see $A$ note on the limit of orbits, Bull. Amer. Math. Soc. vol. 46 (1940) p. 968. Also see Leo Zippin, Transformation groups, in Lectures in topology, University of Michigan Press, 1941, p. 196.

${ }^{9}$ ATW (4.4) p. 248 adapted in F (2.6).

${ }^{10} \mathrm{~F}(2.1)$.

11 ATW (8.1) and (8.2) p. 77.

${ }^{12}$ ATW (4.3) p. 248 adapted in F (2.6). 
and $E_{x}$ the cyclic element of $N$ which contains $x$, then $T^{k}(R) \cdot R=0$ and $T^{k}(x) \in E_{x}$; for otherwise $T^{k}(R)=R$ and $I(k) \cdot R \neq 0$ by the argument used to establish $X$ above. Thus $\bar{K}-N$ is a component-orbit under $T^{k}$ and each component in this finite orbit is bounded by a point of $E_{x}$. If $x \notin X$ then it is possible to construct an $A$-set $M^{*}$ which is a proper subset of $M$ and contains the orbit of $p$. This would be contrary to the definition of $M$. A similar set $M^{*}$ is possible if there is a node of $I(k)$, other than $X$, in $N$. Hence $N+E=C(X, E)=C(X, q)+E$ is the only possibility. Moreover each component of $\bar{K}-N$ has as its boundary a point of $X$; that is, $X=E_{x}$. This suffices to give the structural character of $M$ "near" the "center of rotation" $E$ in $M$; namely $C(X, q)$ and its $k-1$ images extending radially as "spokes" from the "hub" $E$, or a variant of this (when the period of $q$ is less than $k$ ) in which several spokes meet in the same cut point on the orbit of $q$. More specifically, $I(k)=\sum_{n=1}^{k} T^{n}(C(X, q))+E$ and the interior of this $A$-set contains $I(k)-\sum T^{n}(X)$.

Since $I(k)$ contains no point of the orbit of $p$ we may iterate the discussion above using $\bar{K}$ and $T^{k}$ in the roles $M$ and $T$. If $l>k$ is the least positive value of the period function on $K-N$ and $I(l)$ is the set of all elements in $M$ fixed under $T^{l}$, then $l$ is an integral multiple of $k, I(k) \subset I(l)$, and $I(l)$ is an $A$-set. The argument proceeds to show that, when $N^{*}=I(l) \cdot \bar{R}$, we get $N^{*}+X=C\left(X^{*}, X\right)$ where $X^{*}$ is uniquely the node of $I(l)$ in $N^{*}$. Then $I(l)=\sum_{n=1}^{l} T^{n}\left(C\left(X^{*}, q\right)\right)+E$ and this fact, together with results concerning the interior of $I(l)$, are sufficient to introduce the third stage. The third stage introduces the fourth, and so on. When these inductive steps have all been carried out, we find that (as we progress from $E$ toward the orbit of $p$ ) the spoke $C(X, E)$ branches into $l / k$ chains at $X$, these in turn branch at the images of $X^{*}$, and such branching continues indefinitely. This branching occurs only at the designated elements.

The expanding approximation due to Whyburn ${ }^{13}$ (and adapted ${ }^{14}$ to this type of homeomorphism) insures that the above process will envelop all but the end points of $M$, that is, $M-L$. But $\overline{M-L}=M$; and it can be readily shown that $L$ is the closure of the orbit of $p$, hence perfect, one-dimensional, and uncountable. This ends the discussion of Theorem B.

In conclusion it is evident that the proof of Theorem B does not require the full strength of the elementwise periodicity assumption; a study of the general onto-homeomorphism and the subsequent selection of convenient restrictions will clarify this matter. It may also be

${ }^{13}$ ATW (4.7) p. 249.

${ }^{14} \mathrm{~F}(4.2)$. 
remarked that Theorem A may well carry, in such a study, a weight greater than that indicated by its relatively minor role in the proof of Theorem B.

UNIVERSITY OF MISSOURI

\section{THE EQUIVALENCE OF $n$-MEASURE AND LEBESGUE MEASURE IN $E_{n}$}

\section{ARTHUR SARD}

Consider a set $A$ of points in euclidean $n$-space $E_{n}$. For each countable covering $\left\{A_{i}\right\}$ of $A$ by arbitrary sets consider the sum

$$
\sigma=\sum_{i} c_{m} \delta\left(A_{i}\right)^{m}
$$

where $m$ is a fixed positive number, $c_{m}=\pi^{m / 2} / 2^{m} \Gamma[(m+2) / 2]$, and $\delta(A)$ is the diameter of $A$. The constant $c_{m}$ is, for integral $m$, the $m$ volume of a sphere of unit diameter in $E_{m}$. Let $L_{m}(A ; \alpha)$ be the greatest lower bound of all sums $\sigma$ corresponding to coverings for which $\delta\left(A_{i}\right)<\alpha$ for all $i(\alpha>0)$. We define the $m$-measure of $A$ as $L_{m}(A)$ $=\lim _{\alpha \rightarrow 0} L_{m}(A ; \alpha)$. We denote the outer Lebesgue measure of $A$ by $|A|$.

We shall show that $n$-measure and outer Lebesgue measure are equal: $L_{n}(A)=|A|$. A statement on this matter by W. Hurewicz and $\mathrm{H}$. Wallman is true but misleading: these authors assert that $L_{n}(A) / c_{n}$ and $|A|$ may be unequal. ${ }^{1}$

$\mathrm{F}$. Hausdorff has introduced an $m$-measure $L_{m}^{S}(A)$ defined as is $L_{m}(A)$ except that coverings by spheres are used instead of coverings by arbitrary sets. He has shown ${ }^{2}$ that $L_{n}^{S}(A)=|A|$. However $L_{m}(A)$ and $L_{m}^{S}(A)$ are unequal in general, as A. S. Besicovitch has shown ${ }^{3}$ for $m=1, n=2$. S. Saks ${ }^{4}$ and others define $m$-measure as $L_{m}(A) / c_{m}$.

Our proof, which is an obvious extension of Hausdorff's proof, depends on two known theorems.

THEOREM I. Of all sets in $E_{n}$ having a given diameter, the $n$-sphere has the greatest outer Lebesgue measure. ${ }^{5}$

Received by the editors September 23, 1942, and, in revised form, April 2, 1943.

1 W. Hurewicz and H. Wallman, Dimension theory, Princeton, 1941, p. 104.

${ }^{2}$ F. Hausdorff, Dimension und äusseres Mass, Math. Ann. vol. 79 (1919) p. 163.

${ }^{3} \mathrm{~A} . \mathrm{S}$. Besicovitch, On the fundamental geometrical properties of linearly measurable plane sets of points, Math. Ann. vol. 98 (1928) pp. 458-464. R. L. Jeffery, Sets of $k$-extent in n-dimensional space, Trans. Amer. Math. Soc. vol. 35 (1933) p. 634.

${ }^{4}$ S. Saks, Theory of the integral, Warsaw, 1937, pp. 53-54. 